\title{
Methodology to evaluate the risk of vulnerable failure scenarios in a water pipe network
}

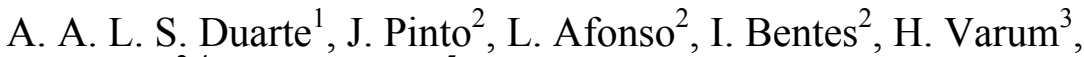 \\ J. Varajão ${ }^{2,4} \&$ J. Agarwal ${ }^{5}$ \\ ${ }^{1}$ Department of Civil Engineering, University of Minho, Portugal \\ ${ }^{2}$ Department of Engineering, ECT, \\ University of Trás-os-Montes e Alto Douro, Portugal \\ ${ }^{3}$ Department of Civil Engineering, University of Aveiro, Portugal \\ ${ }^{4}$ ALGORITMI Research Center, Portugal \\ ${ }^{5}$ Department of Civil Engineering, University of Bristol, UK
}

\begin{abstract}
The emerging theory of vulnerability of water pipe networks intends to give an important contribution for the design of this important component of water supply systems, as well as, for the assessment, rehabilitation and management of the existing ones. The fundamental contribution of this theory is to design water pipe networks more robust since it is able to identify the most vulnerable parts of them. The concept of vulnerability is associated with the disproportionateness of the failure consequences in relation to the initial damage. It is not a theory of robustness. The main objective of this research work is to explain how the risk of a vulnerable failure scenario of a water pipe network may be accessed and how the theory of vulnerability can be relevant.
\end{abstract}

Keywords: risk analysis, water pipe networks, vulnerability, failure scenarios.

\section{Introduction}

The structural vulnerability theory (SVT) has been developed in Bristol University, UK, [1-6]. This theory is able to identify the vulnerable parts of a structure in which the vulnerability concept is related to the possible existence of a disproportionately between failure demand and structural failure consequence. A structure is vulnerable when a small damage demand leads to a 
disproportionately structural failure consequence. The action that may cause that failure can be any type including accidental loads or even human error. The SVT is a theory of structural form and connectivity. Meanwhile, in Agarwal et al. [7], it was proposed and briefly exemplified how the main theoretical concepts of the SVT could be expanded for other types of systems such as electrical, road, organization and water supply networks.

Recent research work has been focused on extrapolating these concepts to the water pipe network (WPN) systems resulting on the emerging theory of vulnerability of water pipe networks (TVWPN) [8-12]. Several research studies have been done in order to predict the probability of the occurrence of failures in WPN (Kleiner and Rajani [13]), and also concerning the vulnerability of WPN based upon fuzzy models (Zidko and Ramos [14]). The water safety plans (WSP) of each water supply system must include safety rules concerning the WPN component. The TVWPN approach may also have an important contribution on the WPN design and also in the assessment, rehabilitation and management processes of the existing ones, mapping its vulnerabilities. The measurement of the scale of damage associated to a failure scenario by the separateness is one particular advantage of the TVWPN application, as a new complement of the classical hydraulic theories.

The main objective of this paper is to propose a methodology able to quantify the risk associated to a vulnerable failure scenario of a WPN. Firstly, the TVWPN is briefly described using a simple WPN example as a support. Secondly, the methodology of quantifying the risk associated to a vulnerable failure scenario of a WPN is delivered. Finally, some conclusions are drawn.

\section{The basic theoretical concepts of the TVWPN}

A system is a set of interacting objects arranged and connected together in some appropriate form. A graph model represents a system in terms of nodes and links. In a WPN system nodes are the joints and links are the pipelines. A cluster is a subset of the graph model in which the objects are in some sense more tightly connected to each other than to other objects outside of the cluster. A WPN cluster is considered as being a subset of the WPN in which the pipelines are in some sense more tightly connected to each other than the other pipe lines outside of the WPN cluster. A WPN ring is assumed of being a maximum of two connected WPN clusters which can supply water between different points. A $W P N$ leaf cluster (or WPN primitive cluster) contains a single pipeline and adjacent joints. A WPN branch cluster is a cluster that contains more than one leaf cluster; a sub-WPN is a branch cluster. A WPN ring represents a WPN branch cluster. The WPN reference cluster is the storage tank which is the cluster from which the WPN is separated for the total failure scenario to occur. The $W P N$ root cluster contains the entire WPN including the reference cluster. A WPN deteriorating event may be the damage that results from any type of action which causes the loss, by a WPN ring, of the capacity to supply good quality of water between points, it may be the collapse of a pipeline, an obstruction or even, but extremely important, the degradation of water quality. Thus, a WPN 
vulnerable failure scenario is considered as being an ordered sequence of WPN deteriorating events by which the performance of a WPN decreases.

The well formedness of a WPN cluster has been assumed of being the measure of the quality of the form of a WPN which is independent of the coordinate system. In some way, it has to be related to the stiffness of the pipelines, the type of the joint, the configuration of the WPN and the connection between the pipelines (Pinto et al. [10]). It has been assumed that the total head losses $\left(\Delta H_{T}\right)$, given by eqn. (1), is a good indicator of the well formedness of a WPN because it includes indirectly all the above factors. A small total head loss indicates a good form of a WPN branch cluster.

$$
\Delta H_{T}=\sum_{j=1}^{p} \Delta H_{j}+\sum_{i=1}^{u} \Delta H_{L_{i}}
$$

where $\Delta H_{T}$ is the total head losses, the $\Delta H$ is the occurred continuous head losses in the $j$ pipeline, $\Delta H_{L}$ is the local loss, $p$ is the total number of the existing pipelines in a WPN branch cluster and $u$ is the total number of singularities existing in a WPN branch cluster.

The nodal connectivity $(\eta)$ of a WPN branch cluster measures the connection of that WPN branch cluster and the rest of the WPN or, in other words, the existing alternative water supplying paths between that WPN branch cluster and the rest of the WPN. It also represents how ease a WPN branch cluster forms WPN rings with the others branch clusters of the rest of the WPN. At this stage, the $\eta$ of a WPN branch cluster is the sum of the all pipelines, outside of the cluster, that converge to the joints of that WPN branch cluster.

The $W P N$ damage demand $(E)$ has been considered, at this stage, as being a measure of the effort required to cause a deteriorating event in the WPN which is proportional to the strength capacity of a pipeline. Thus, it has been assumed that $P s$ is the service pressure. The WPN relative damage demand $\left(E_{r}\right)$ of a WPN failure scenario is the ratio of the WPN damage demand $(E)$ of a failure scenario to the maximum possible damage demand of a failures scenario in the WPN system $\left(E_{\max }\right)$ (i.e. a failure scenario in which deteriorating events occur in every WPN primitive clusters).

The separateness $\left(\gamma_{r}\right)$ has been defined as a measure of the failure consequence and has been calculated as the ratio of the loss in WPN well formedeness of the deteriorated WPN to the well formedeness of the intact WPN. Total separateness occurs when the WPN system becomes disconnected from the reference cluster.

The WPN vulnerability index $(\varphi)$ of a failure scenario is a measure of the vulnerability of a WPN and it can be measured by the ratio of the separateness $\left(\gamma_{r}\right)$ to the relative damage demand $\left(E_{r}\right)$. It is a measure of the disproportionality of the failure consequence (separateness) to the damage (damage demand) required for that failure. An expressive value of $\varphi$ related to a certain failure scenario indicates that the WPN shows signs of high vulnerability because there is a disproportionate relation between the extension of the deterioration of the WPN and the effort required to cause that damage. 


\section{Application of the TVWPN}

The application of the TVWPN to a WPN consists of two main stages which are the clustering and the unzipping processes.

In brief, the clustering process consists on a progressive formation of WPN branch clusters that are tightly connected, starting at the first level by only using primitive clusters (pipelines) and finishing, at the last level, by having the whole WPN, including the storage tank, completely agglutinated (resulting in a root WPN branch cluster). It is a selective process that requires criteria which are identified following.

Based upon the information resulted from the clustering process it is possible to build the hierarchical model of the WPN which is an abstract way of representing the WPN in a interconnected and well formedness bases. It is by this model that the vulnerable failure scenarios are identified through the unzipping process. The unzipping process is the last stage of the application of the TVWPN. Unzipping the hierarchical model from the top to the bottom and using criteria, WPN deteriorating events are found with the purpose of identifying vulnerable failure scenarios. These vulnerable failure scenarios allow than to identify the parts of the WPN which are more vulnerable.

\subsection{Clustering process}

The clustering process is a progressive and a selective process which consists of identifying WPN rings made up of joints and pipelines at the first and familiar level of definition of a WPN. A new set of WPN rings of clusters is then formed to provide a second level of definition of the WPN. The process of clustering is repeated to form even higher levels of definition in a hierarchy until a single WPN cluster, the whole WPN including the storage tank, remains. This process uses five clustering criteria which are applied to decide the next WPN branch cluster to be formed in each level of definition. These five clustering criteria are in order the following: a) the minimum total head loss, $\Delta H_{T m i n}$; b) the maximum damage demand, $E_{\max }$; c) the maximum nodal connectivity, $\eta_{\max }$; d) the maximum distance from the storage tank, $D_{i \text { Smax }}$; e) free choice, $F_{C}$.

The hierarchical model is another alternative graphical way of representing a WPN. However, the WPN elements (pipes and fittings) appear in that model rearranged according to the quality of the form of the WPN and resulted from the previously applied clustering process. The interpretation of a hierarchical model has to start from the bottom to the top. Going up from the bottom to the top of the hierarchical model, the new WPN branch clusters and the respective primitive used clusters are clearly identified. Simultaneously, the WPN rings that represent these new WPN branch clusters and the applied clustering criteria used for the candidate selection are also shown. The part of the WPN that is represented in the bottom of the hierarchical model has better form than the others parts that are represented above. This model is extremely important for the last stage of the application of the TVWPN, the unzipping process, and as it will be detailed explained during the following section. 


\subsection{The unzipping process}

The unzipping process is the second and the last stage of the application of the TVWPN which uses the hierarchical model of a WPN as the base for searching the vulnerable WPN failure scenarios. The hierarchical model is unzipped from the top to the bottom focusing on all the existing WPN branch clusters. Each WPN branch is unzipped in turn and deteriorating events are found until that WPN branch cluster or the whole WPN becomes totally inoperative. Meanwhile, when a deteriorating event is found, the WPN branch cluster changes and, therefore, it is required to cluster and to define the respective new hierarchical model of the changed WPN branch cluster. This fact indicates that the unzipping process is an iterative process. The ordered sequence of deteriorating events resulted by this process define the vulnerable failure scenarios found by the TVWPN. For the search of deteriorating events, the unzipping process also uses criteria (unzipping criteria). The unzipping criteria are, by order of application, the following: a) it is not the reference cluster (storage tank), $N_{R}$; b) it connects directly to the reference cluster, $C_{D} ; \mathrm{c}$ ) it is a leaf cluster (or a primitive cluster or a pipeline) rather than a WPN branch cluster, $L_{C}$; d) it has the higher value of head loss, $S_{\Delta H}$; e) it has the smallest damage demand, $S_{E} ;$ f) it was clustered the latest, $\left.C_{L} ; \mathrm{g}\right)$ free choice, $F_{C}$.

At this research stage, the storage tank has been considered as being undamaged, as a simplification. If a pipeline connects directly to the storage tank, then it is likely that its service loss will result in a disproportional failure consequence of the WPN. It is possible to identify a deteriorating event in a leaf cluster (i.e. a pipeline) rather than a branch cluster.

\subsection{Vulnerable failure scenarios}

Through the application of the TVWPN it is possible to identify the following vulnerable failure scenarios:

a) The total failure scenario is the one where least effort is required for the whole WPN to become inoperative (there is no water supplying to any point and the whole WPN becomes disconnected from the storage tank). Among the vulnerable failure scenarios identified having separateness equal to 1 , the total failure scenario is the one that has higher value of vulnerability index $(\varphi)$;

b) The maximum failure scenario is the one which results in maximum damage from least effort and it is not necessary the total. Among the vulnerable failure scenarios found the maximum failure scenario is the one with highest value of $\varphi$. The maximum failure scenario is related to the most vulnerable part of a WPN;

c) The minimum failure scenario is related to the worst well formed part of a WPN and, in generally, corresponds to the last leaf cluster to be clustered in the clustering process;

d) The minimum demand failure scenario is related to the weakest part of a WPN to suffer damage. Corresponds to the leaf cluster that has the smallest value of damage demand;

e) The interesting failure scenario is the one in which the designer is specifically interested for local reasons such as sensitivity to particular usage. 


\section{An example of the application of the TVWPN}

In order to exemplify the application of the TVWPN and to complement the description of the theoretical concepts of this theory the WPN of Figure 1 is used. It is made of four pipes, four joints and one storage tank.

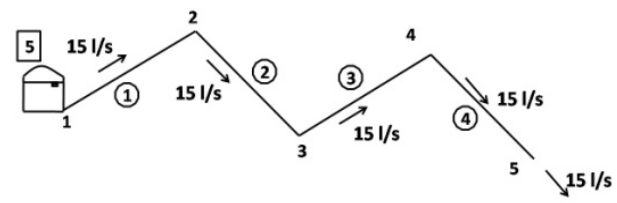

Figure 1: $\quad$ WPN used as an example.

Meanwhile, Table 1 summarises some geometrical and hydraulic measures of the WPN. The material for all the pipelines is polyvinyl chloride (PVC) with $0.01 \mathrm{~mm}$ roughness and $0.6 \mathrm{MPa}$ service pressure is assumed. The relevant geometric (pipe's length and diameter) and hydraulic (flow and head loss) data of the WPN is presented in Table 1.

Table 1: $\quad$ Geometric and hydraulic data of the WPN.

\begin{tabular}{|c|c|c|c|c|c|}
\hline Leaf cluster & Joints & Length $(\mathrm{m})$ & Flow $(1 / \mathrm{s})$ & Diameter $(\mathrm{mm})$ & Head loss $(\mathrm{m})$ \\
\hline 1 & $1 ; 2$ & 200 & 15 & 160 & 0.84 \\
\hline 2 & $2 ; 3$ & 200 & 15 & 160 & 0.84 \\
\hline 3 & $3 ; 4$ & 200 & 15 & 160 & 0.84 \\
\hline 4 & $4 ; 5$ & 200 & 15 & 160 & 0.84 \\
\hline
\end{tabular}

The flow and the head loss values result from the hydraulic design of the WPN which it is not presented here for simplification. Table 2 shows the two main plots (position and pressure) of the specific energy (Bernoulli trinomial) at the several network nodes.

Table 2: $\quad$ Piezometric parameters at the WPN nodes.

\begin{tabular}{|c|c|c|c|c|c|c|}
\hline \multirow{2}{*}{$\begin{array}{c}\text { Leaf } \\
\text { cluster }\end{array}$} & \multicolumn{2}{|c|}{ Topographical elevation $(\mathrm{m})$} & \multicolumn{2}{c|}{ Piezometric elevation $(\mathrm{m})$} & \multicolumn{2}{c|}{ Piezometric height $(\mathrm{m})$} \\
\cline { 2 - 7 } & Upstream & Downstream & Upstream & Downstream & Upstream & Downstream \\
\hline 1 & 163.68 & 130.00 & 293.68 & 165.84 & 3.00 & 35.84 \\
\hline 2 & 130.00 & 140.00 & 270.00 & 165.00 & 35.84 & 25.00 \\
\hline 3 & 140.00 & 130.00 & 270.00 & 164.16 & 25.00 & 34.16 \\
\hline 4 & 130.00 & 135.00 & 265.00 & 163.32 & 34.16 & 28.32 \\
\hline
\end{tabular}

The clustering process is the first stage of the application of the TVWPN. Table 3 summarises the four steps required for the WPN used as an example to become a single branch cluster, branch cluster 9. Each step includes the identification of possible candidates (leafs or branches) to be clustered (Figure 2). For each element of the WPN candidates the $\Delta H_{T}, E, \eta$ and $D_{i S}$ are calculated. 
Table 3: $\quad$ Steps of the clustering process applied in the presented WPN.

\begin{tabular}{|c|c|c|c|c|c|c|}
\hline \multirow{2}{*}{ Step } & $\begin{array}{c}\text { Branch cluster } \\
\text { candidate }\end{array}$ & $\begin{array}{c}\Delta H_{T} \\
(\mathrm{~m})\end{array}$ & $F_{\boldsymbol{s}}(\mathrm{MPa})$ & $\eta$ & $\begin{array}{c}\text { Dis } \\
(\mathrm{m})\end{array}$ & Formed branch cluster \\
\hline \multirow{3}{*}{$1^{\text {st }}$} & $1+2$ & 1.68 & 1.2 & 1 & 0 & - \\
\cline { 2 - 7 } & $2+3$ & 1.68 & 1.2 & 2 & 200 & 6 \\
\cline { 2 - 7 } & $3+4$ & 1.68 & 1.2 & 1 & 400 & - \\
\hline \multirow{2}{*}{$2^{\text {nd }}$} & $1+6$ & 2.52 & 1.8 & 1 & 0 & - \\
\cline { 2 - 7 } & $4+6$ & 2.52 & 1.8 & 1 & 200 & 7 \\
\hline \multirow{2}{*}{$3^{\text {rd }}$} & $1+7$ & 3.36 & 2.4 & 0 & 0 & 8 \\
\hline $4^{\text {th }}$ & $5+8$ & - & - & - & - & 9 \\
\hline
\end{tabular}
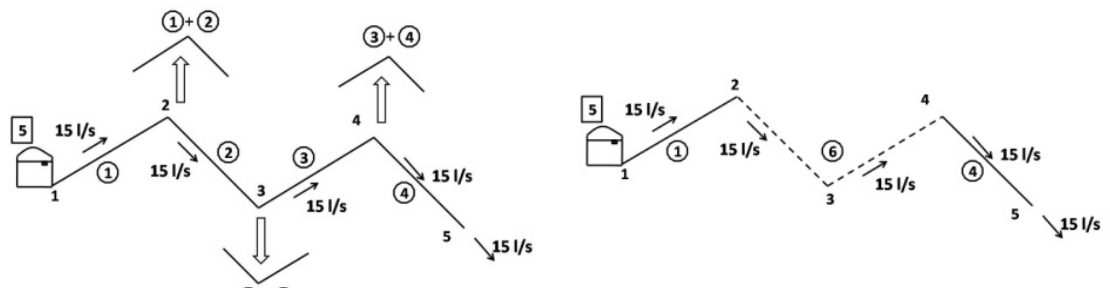

(2) + (3)

a) $1^{\text {st }}$ step: Forming WPN branch cluster 6
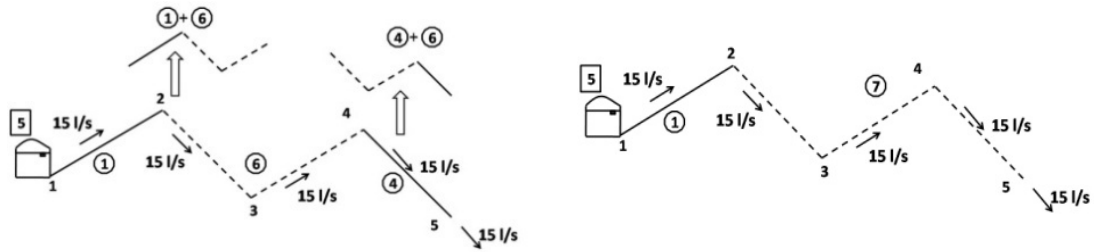

b) $2^{\text {nd }}$ step: Forming WPN branch cluster 7
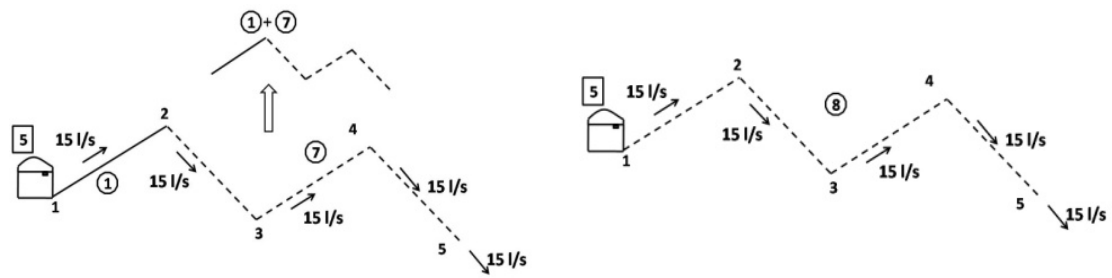

c) $3^{\text {rd }}$ step: Forming WPN branch cluster 8

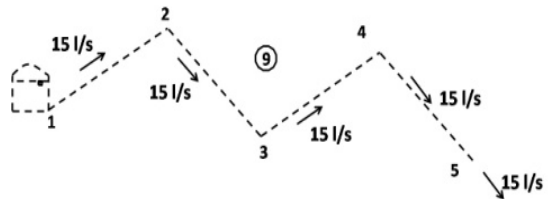

d) $4^{\text {th }}$ step: Forming WPN branch cluster 9

Figure 2: Clustering process. 
Using the five clustering criteria the better well formed candidate is selected. For instance, in the first step there are three candidates to be clustered $(1+2,2+3$ and 3+4) (Figure 2a)). Among these candidates, the candidate $2+3$ is selected to form a branch cluster because it has the higher value of $\eta$ (the maximum nodal connectivity $\left(\eta_{\max }\right)$ was the clustering criterion applied to make a decision). The branch cluster 6 is formed and its children are leaf clusters 2 and 3. During the second step there are two candidates to form a new branch cluster which are $1+6$ and 4+6 (Figure 2b)). The applied clustering criteria was the maximum distance from the storage tank $\left(D_{i S \max }\right)$ and the branch cluster 7 was formed which has leaf cluster 4 and branch cluster 6 as children. There is only a single candidate to be clustered in the third step which is cluster leaf cluster 1 and branch cluster 7, (Figure 2c)). The branch cluster 8 is formed. The same feature occurs in the last step of the clustering process of this example, in where remained candidate to be clustered is the reference cluster 5 and branch cluster 8 (Figure 2d)). At this stage, the WPN is totally clustered (branch cluster 9) and the clustering process ends. This example has the particularity of showing the application of different clustering criteria contrasting with the previously reported examples $[10,11]$. Based on the information resulted from the clustering process it is possible to draw the hierarchical model of the WPN used as an example, Figure 3. There, the leaf, reference and branch clusters are clearly differentiated. The associated WPN ring representing each branch cluster and the applied clustering criterion are also indicated. Since the WPN used as an example is simple and corresponds to a series of pipes, the WPN rings are opened.

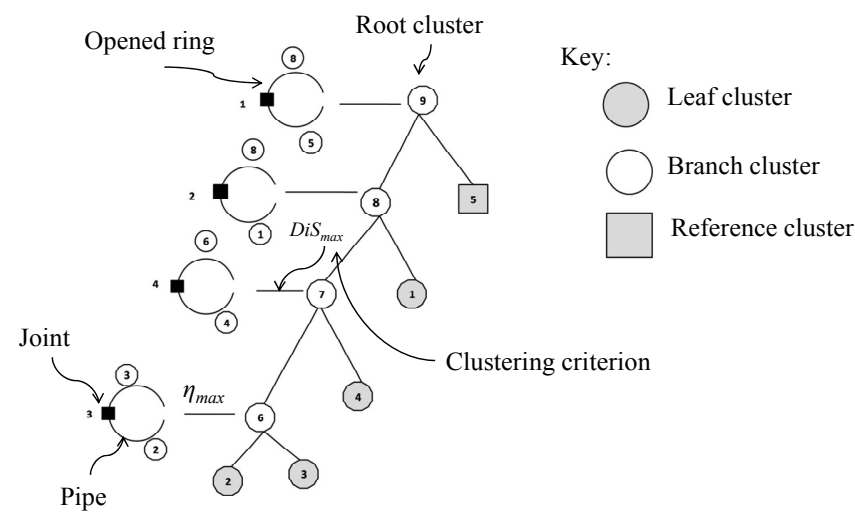

Figure 3: Hierarchical model of the presented WPN example.

The second and the last stage of the application of the TVWPN is the unzipping process and it is described from Figure 4 to Figure 6 . All the branch clusters are unzipped, in turn, and the application of the unzipping criteria traces the unzipping path which ends up by identifying the leaf cluster to suffer damage (a deteriorating event). An order sequence of deteriorating events is a vulnerable failure scenario. In this example, the identified vulnerable failure scenarios are only related to a sequence of one deteriorating event (Table 4). 

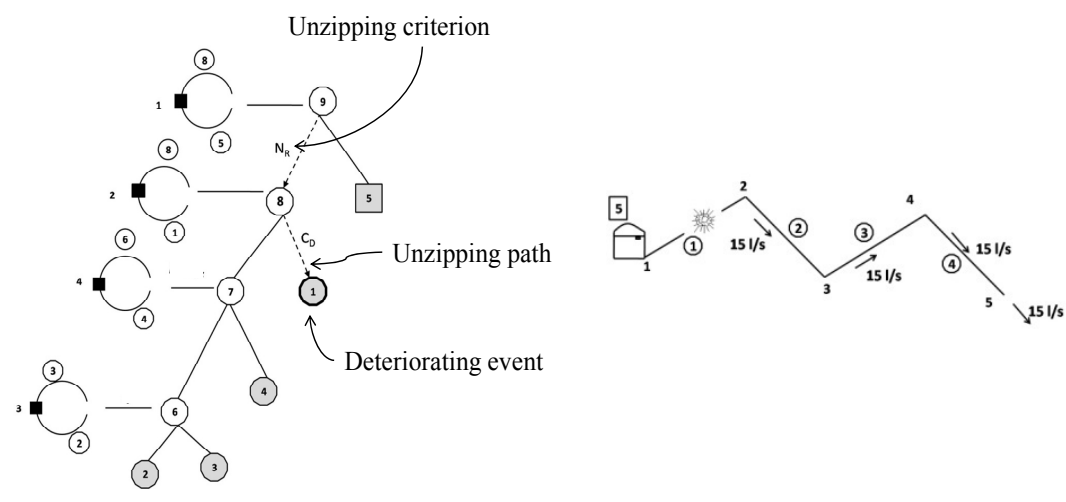

a) Unzipping path

b) Deteriorating event $\mathrm{T} 1$

Figure 4: $\quad$ Unzipping of branch cluster 9.

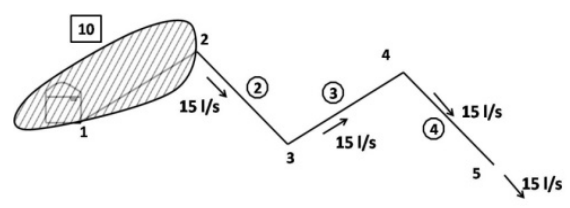

a) Branch cluster 7

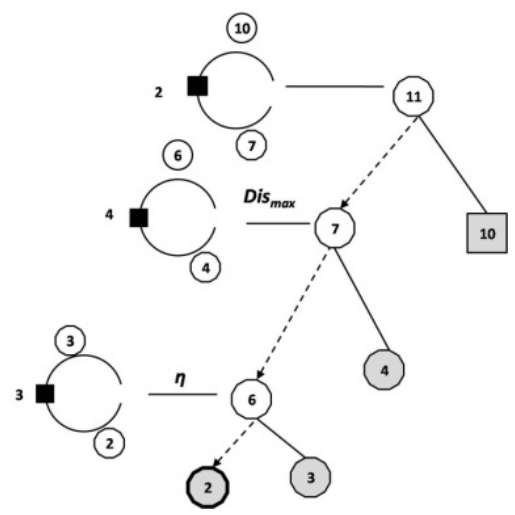

c) Unzipping path

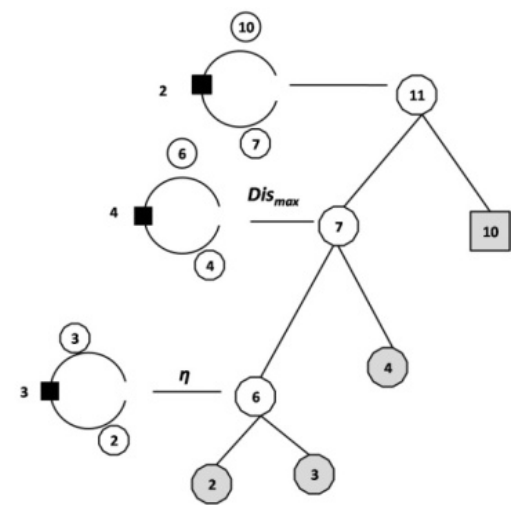

b) Hierarchical model of branch cluster 7

Figure 5: $\quad$ Unzipping of branch cluster 7. 


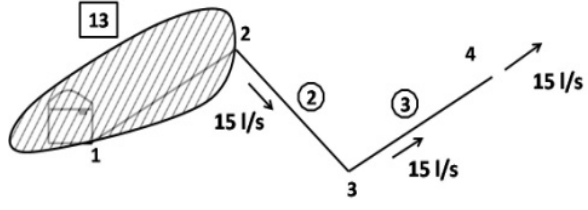

a) Branch cluster 6

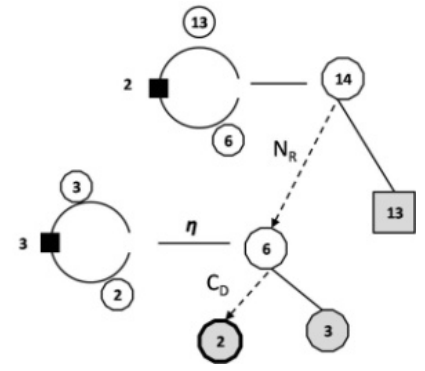

c) Unzipping path

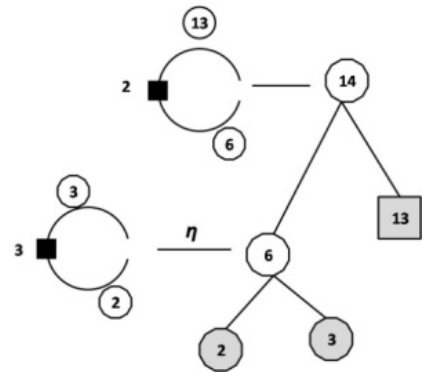

b) Hierarchical model of branch cluster 6

Equal to Figure 5d)

Figure 6: $\quad$ Unzipping of branch cluster 6.

Table 4: Vulnerable failure scenarios identified through the unzipping process.

\begin{tabular}{|c|c|c|c|c|}
\hline & Vulnerable failure scenario & $\bar{\gamma}_{r}$ & $E_{R}$ & $\varphi$ \\
\hline 1 & $\mathrm{~T} 1$ & 1 & 0.250 & 4 \\
\hline 2 & $\mathrm{~T} 2$ & 0.750 & 0.250 & 3 \\
\hline
\end{tabular}

Table 4 also includes the vulnerable parameters $\left(\bar{\gamma}_{r}, E_{R}\right.$ and $\left.\varphi\right)$ associated to each vulnerable failure scenarios, which are fundamental to identify the specific vulnerable failure scenarios. Thus, according to the description given in section 3.3, Figure 2 or 3 and the vulnerable parameters of Table 4 the total, the maximum, the minimum and the minimum demand failure scenarios are the same, to damage pipe $1(\mathrm{~T} 1)$. This fact is a coincidence rather than a rule and occurs because this vulnerable failure scenario has simultaneously $\bar{\gamma}_{r}$ equal to one, maximum value of $\varphi$, all the pipes have the same value of $E$ and it was the latest to be clustered.

This result gives additional relevance to this example. It is without doubt that the area of the WPN used as an example related to pipe 1 presents an undesirable vulnerability which may suggest that a mitigation analysis should be required in order to reduce this weakness of the WPN. 


\section{Risk of a WPN vulnerable failure}

Based on [15-17], risk has been defined as the combined effect of the chances of occurrence of some failure or disaster and its consequences, in a given context, can be estimated by the eqn. (2).

$$
R(\text { Context })=p \times \text { Con }
$$

where $R$ (Context) is the risk of a failure in a given context, $p$ is the chance of a failure and Con is the consequence of a failure.

According to Pinto et al. [6], within the totality of all possible modes of failure (including business and financial failure) the structural risk $(S R)$ has been defined as one aspect (structural functionality) of the total project risk, given by eqn. (3):

$$
S R=p f s \times S c o n
$$

where, $p f_{s}$ is the probability of failure which is calculated using the techniques of structural reliability analysis and SCon is a function of structural separateness. In the WPN context, the associated WPN risk (WR) may be defined by eqn. (4):

$$
W R=p f w \times W c o n
$$

where, $p f w$ is the probability of WPN failure and WCon may be a function of the WPN separateness.

\section{Conclusions}

The theory of the vulnerability of water pipe networks (TVWPN) was briefly described. The main purposed of the TVWPN is to identify the most vulnerable parts of water pipe networks (WPN) and consequently to increase robustness of this type of system.

A WPN example of four pipes was used for the sake of simplicity. However, this example has shown to be very interesting because it allows figuring out the relevance of the clustering criteria defined in this theory.

Furthermore, this example also gives a peculiar result because the total, the maximum, the minimum and the minimum demand failure scenarios are both the same. This fact is a coincidence rather than a rule.

This theory may be applied in the design, maintenance and management of the WPN systems. This theory also gives a measure of scale of a failure consequence by the separateness concept.

This information may be extremely helpful for the quantification of the risk associated to a WPN failure.

\section{References}

[1] $\mathrm{Lu} \mathrm{Z.,} \mathrm{Yu} \mathrm{Y.,} \mathrm{Woodman} \mathrm{N.J.,} \mathrm{Blockley} \mathrm{D.I.,} \mathrm{A} \mathrm{theory} \mathrm{of} \mathrm{structural}$ vulnerability. Journal the Structural Engineer, 77(18), pp. 17-24 , 1999. 
[2] Yu Y., Analysis of structural vulnerability. PhD Thesis, University of Bristol, UK, 1997.

[3] Lu Z., Structural vulnerability analysis. PhD Thesis, University of Bristol, UK, 1998.

[4] Agarwal J., Blockley D.I., Woodman N.J., Vulnerability of 3-dimensional trusses. Structural Safety, 23(3), pp. 203-220, 2001.

[5] Pinto J.T., The risk of a vulnerable scenario. PhD Thesis, University of Bristol, UK, 2002.

[6] Pinto J.T., Blockley D.I., Woodman N.J., The risk of vulnerable failure. Structural Safety, 24(2-4), pp. 107-122, 2001.

[7] Agarwal J., Blockley D.I., Woodman N.J., Vulnerability of systems. Civil Engineering Environ. Systems, 18(2), pp. 141-165, 2001.

[8] Pereira L., Varajão J., Pinto T., Bentes I., Introduction to water supply network vulnerability theory (TVRHAA). Proc. of the ENEG 2009 Sustainability in water urban cycle, ed. APDDA (CD-Rom), Lisbon, Portugal, November 2009. (in Portuguese)

[9] Bastos C., Duarte A.A.L.S., Bentes I., Pinto J.T., Theory of Vulnerability of water supply network (TVRHAA). Proc. of the $9^{\text {th }}$ Symposium of Hydraulics and Water Resources of Portuguese Spoken Countries (SILUSBA), ed. APRH (Cd-Rom), Benguela, Angola, October 2009. (in Portuguese)

[10] Pinto, J.T., Varum, H., Bentes, I., Agarwal, J., A Theory of Vulnerability of Water Pipe Network (TVWPN). Water Resources Management. Online. www.springerlink.com/content/x024016077428716/, 2010

[11] Pinto J., Afonso L., Varajão J., Bentes I., Varum H., Duarte A.A.L.S., Agarwal J., Increasing the quality of water pipe network systems by tracing the vulnerability. Proc. of Advances in Waste Management, eds: A. Kallel, A. Hassairi, C. Bulucea, N. Mastorakis, Sousse, Tunisia, May 3-6, 2010.

[12] Pinto J., Varum H., Bentes I., Contribution to the study of the vulnerability of water supply network. Proc. of Engineering 2009 - Innovation and Development, UBI, Covilhã, Portugal, November 2009. (in Portuguese)

[13] Kleiner Y. \& Rajani B., Considering time-dependent factors in the statistical prediction of water main breaks. Proc. of American Water Works Association: Infrastructure Conference, Baltimore, Maryland, March 1215. pp 1-12, 2000.

[14] Zidko V. \& Ramos H., Fuzzy model in the vulnerability assessment of water supply systems. Recursos, 30(1), pp. 5-25, 2009.

[15] Blockley DI, Godfrey P., Doing it differently. Thomas Telford; 2000.

[16] Agarwal J., Blockley D.I., Woodman N.J., Improving System's Dependability. Advances in Safety and Reliability. Proc. of ESREL '97, Lisbon, pp. 2329-2337, 1997.

[17] Blockley D.I., Risk based structural safety methods in context. Structural Safety, 21(4), pp. 335-348, 1999. 